\title{
Sparse Unbiased Analysis of Anatomical Variance in Longitudinal Imaging
}

\author{
Brian Avants, Philip A. Cook, Corey McMillan, Murray Grossman, \\ Nicholas J. Tustison, Yuanjie Zheng, and James C. Gee \\ Depts. of Radiology and Neurology \\ University of Pennsylvania \\ Philadelphia, PA 19104-6389 \\ avants@grasp.cis. upenn. edu
}

\begin{abstract}
We present a new algorithm for reliable, unbiased, multivariate longitudinal analysis of cortical and white matter atrophy rates with penalized statistical methods. The pipeline uses a step-wise approach to transform and personalize template information first to a single-subject template (SST) and then to the individual's time series data. The first stream of information flows from group template to the SST; the second flows from the SST to the individual time-points and provides unbiased, prior-based segmentation and measurement of cortical thickness. MRI-bias correction, consistent longitudinal segmentation, cortical parcellation and cortical thickness estimation are all based on strong use of the subject-specific priors built from initial diffeomorphic mapping between the SST and optimal group template. We evaluate our approach with both testretest data and with application to a driving biological problem. We use testretest data to show that this approach produces (a) zero change when the retest data contains the same image content as the test data and (b) produces normally distributed, low variance estimates of thickness change centered at zero when test-retest data is collected near in time to test data. We also show that our approach—when combined with sparse canonical correlation analysis-reveals plausible, significant, annualized decline in cortical thickness and white matter volume when contrasting frontotemporal dementia and normal aging.
\end{abstract}

\section{Introduction}

Longitudinal MRI studies are often underpowered due to the challenges of data collection, data consistency and data analysis. One of the most difficult detection problems in longitudinal neuroimaging is tracking cortical thickness reduction in dementia via clinical resolution T1 MRI. These images typically have resolution near $1 \mathrm{~mm}^{3}$ whereas the cortex itself is only one to five millimeters thick in this age range and thinner in diseased regions. Power is also reduced by the fact that these studies are testing for "differences of differences," which increases noise in the measurement and reduces power. Additionally, there are few standard pipelines that exist for processing longitudinal thickness data. Finally, the combined challenges of segmentation, registration and change estimation make longitudinal pipelines difficult to validate. For these reasons, the majority of studies have focused on whole brain or regional, lobe-based methods for 
estimating longitudinal atrophy rates such as the Brain Boundary Shift Integral (BBSI) $\left[\begin{array}{l|l|l|l|l}9 & 13 & 12 & 8 & 6\end{array}\right]$. These methods gain stability by integrating over large regions, but lose specificity. Other studies have increased power by using very large cohorts. Shaw, et al. used 1133 images to study differences in cortical thickness change between ADHD and controls [10]. However, studies of rare dementia do not have the luxury of large cohorts and thus few groups have attempted voxel or surface-wise estimates of longitudinal cortical thickness group differences.

Power and accuracy can be increased in longitudinal studies by combining unbiased estimation with feature selection. Yushkevich et al. showed that distributing interpolation equally across all of a subject's images is critical for eliminating the bias associated with longitudinal hippocampal atrophy [14]. This leads to a basic principal in longitudinal studies: all images should undergo an equivalent number of interpolations. While this principal increases accuracy, precision remains relatively low due to the noise associated with these studies. To alleviate this concern, multivariate feature selection may be used to not only reduce the multiple comparisons problem in a principled way [1] but also select a subset of data that contains strong signal that agrees across two independent views of the data. Sparse canonical correlation analysis allows one to select a controllably sparse, mutually informative subset of voxels from paired, complementary sets of measurements taken on the same subject. This subset of voxels may be selected without reference to diagnosis. Thus, after selected, they may be passed to a test for group differences without additional statistical penalty or circularity [7].

Here, we develop a new multivariate, morphometric framework — entitled Sparse Unbiased Analysis of Anatomical Variance (SUAAV) - for detecting voxel-wise group differences in cortical thickness and white matter atrophy rates. SUAAV builds on existing, open-source image registration, MRI-bias correction, segmentation, cortical thickness and sparse estimation tools. We apply and evaluate SUAAV in the challenging problem of using serial T1-weighted MRI to contrast rates of atrophy in cortical thickness and white matter volume between frontotemporal dementia (FTD) and a small set of control data. Tremendous interest exists in sensitive, unbiased approaches for quantifying longitudinal thickness change as such methods are essential tools in treatment trials. SUAAV is designed specifically for these types of tracking studies and achieves power via variable selection and accuracy via application of the latest knowledge in unbiased implementation of image measurement tools.

The most important novel components of SUAAV include: (1) unbiased segmentation based on propagation of tissue priors from a group template, to a single-subject template and, finally, to each time point image; (2) unbiased estimation of cortical thickness change from in vivo resolution T1 MRI; (3) use of multivariate feature selection to increase sensitivity in longitudinal studies of correlated white matter and cortical thickness change. The framework, though advanced, has relatively few requirements in that it only requires a prior-labeled template and serial data where two complementary views/images of each subject's time change are available. In this study, we derive these measures from a single modality, clinical T1-weighted imaging, by pairing an estimate of annualized cortical thickness change with annualized white matter volume change. We evaluate SUAAV with test-retest data and show that results are biologically plausible and sensitive in real clinical data. 


\section{Methods}

\subsection{Subjects and Imaging}

The input data to our analysis consists of serial T1-weighted MRI collected longitudinally on a Siemens 3T scanner. Each study began with a rapid sagittal T1-weighted scan to determine patient position. A T1-weighted structural acquisition was then acquired with TR $($ repetition time $)=1620 \mathrm{~ms}$, TE $($ echo time $)=3 \mathrm{~ms}$, slice thickness: 1 mm, in-plane resolution: $.9766 \mathrm{~mm} \times .9766 \mathrm{~mm}$ and field of view (FOV) $256 \times 256 \times$ 192. All data were checked for adequate quality across time points before proceeding to the analysis. An experienced neurologist, who specializes in discrimination of these diseases, grouped the subjects into likely syndromes (-) based on clinical phenotype and cognitive testing. Our analysis included 7 elderly control subjects and 26 FTD subjects matched for sex, age and education. All subjects' follow-up images were acquired approximately one year after the initial visit and there was no siginficant difference in the set of delay times between the control and FTD longitudinal image sets.

\subsection{Sparse Unbiased Analysis of Anatomical Variance}

We now describe the components of the SUAAV framework for gaining unbiased estimation of longitudinal neurodegeneration in white matter and cortical thickness and shown in figure 1 and figure 2
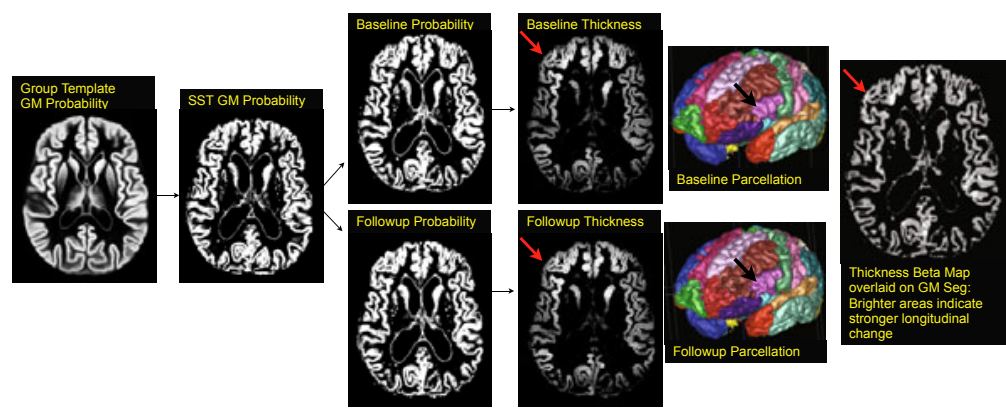

Fig. 1. The SUAAV algorithm's pipeline requires a template that contains cortical priors and cortical labels that are used in a parcellation scheme. These priors are mapped first to a single-subject template (SST) and used to initialize segmentation of the SST. The resulting probability maps are then deformed from SST space to individual space to initialize a prior-constrained segmentation of (in this case) a baseline and follow-up image collected at a one year interval. Thickness maps and cortical parcellation are then computed for each time point image. While parcellation is not explicitly required by SUAAV, we use parcellation, here, to evaluate the stability of the pipeline in test-retest data and to verify the findings provided by the voxel-wise analysis. Red arrows point to a region associated - in the group analysis—-with significant atrophy and black arrows point to the corresponding region in the parcellation. 
1. SST Initialization and Optimization: We construct an optimal, unbiased withinsubject template from each subject's serial data by rigidly aligning the first time point image to the group template. Subsequent time points are aligned to the first time point. The result is then averaged to produce an unbiased initialization. Given the initialization, we use the method described in [1], and available in the ANTS toolkit [4], to compute the optimal shape and appearance SST with respect to all time points. This approach guarantees that the mapping for each subject to the group template or to the SST involves the same number of interpolations (one) or compositions (affine + diffeomorphism to go to the SST and affine + diffeomorphism + affine + diffeomorphism to go to the group template).

2. Mapping to Group Template and Tissue Priors: Given the SST from the first step, we use standard correlation-based ANTS diffeomorphic registration to find the invertible mapping between the SST and template. This mapping then allows us to deform tissue-priors for cerebrospinal fluid, gray matter and white matter to the SST-space. The white matter tissue probability map is then passed to the N4 algorithm [11] which uses this map to perform a weighted B-Spline based bias correction. The bias corrected image, along with tissue priors, are then passed to a prior-based, parametric Expectation-Maximization, Markov-Random-Field segmentation algorithm that fits the models to the SST. Here, the prior is used only for initialization of the EM-MRF algorithm.

3. Time Point Segmentation: The segmentation results in the SST space are deformed from the SST to each individual time point, based on the maps that were computed during the SST optimization. The same segmentation procedure from step 2 is then performed for each time point, but, here, the prior terms are maintained during optimization of the EM-MRF to prevent a large change from the initialization. Given the cortical segmentation, we also parcellate the cortex using the method described in [5], which deforms a template parcellation to the subject and fits the initial parcellation to the cortical data by using the fast marching method.
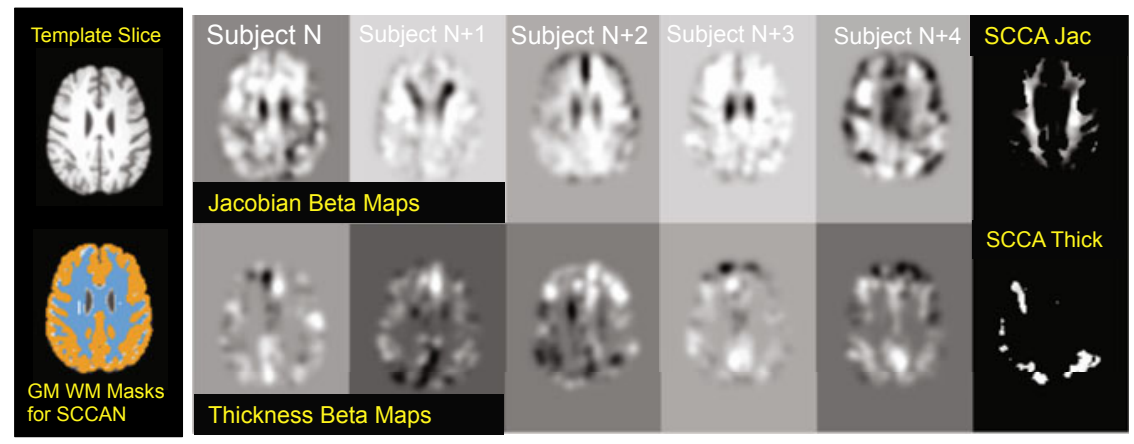

Fig. 2. The SCCAN component of SUAAV is illustrated. The template and the masks for each "view" (Jacobian and thickness change) are at left. The top row shows a selection of individual Jacobian change images in the template space, after smoothing. The same subject's thickness change images, after smoothing, are in the bottom row. A slice from the SCCAN weight maps for each view is shown at right. 
The original template parcellation is based on the NIREP data (www.nirep.org). This approach was shown to perform as well as Freesurfer cortical parcellation within the volumetric domain [5].

4. Thickness Estimation: Given the segmentation results for each time point from step 3, we measure the cortical thickness at each time point with Diffeomorphic Registration Based Cortical Thickness (DiReCT) [2].

5. Annualized Atrophy: We map the resulting cortical thickness images back to the single-subject template and estimate longitudinal change in cortical mm/yearwithin the SST gray matter mask - by using the beta parameter in a linear regression fit, that is, change in thickness $\approx x_{0}+\beta_{T} * t$ where $x_{0}$ is the intercept and $t$ is the time parameter. Solving this regression problem at every voxel provides an image $\beta_{T}(\mathbf{x})$. When only two time points are present, this image can be computed by subtracting the baseline image from the followup image directly and dividing by the time separation. In the same way, but within the SST white matter mask, we estimate white matter volume change from the Jacobian of the serial deformation in SST space, providing image $\beta_{J}(\mathbf{x})$. We median filter both $\beta_{J, T}(\mathbf{x})$ images to remove few-voxel registration/interpolation inaccuracies. We then map both of the time-change beta maps (thickness and Jacobian) for each subject to the group template space at two millimeters.

6. Feature Selection/Dimensionality Reduction: Once the above steps are completed for all subjects, we locate reliable regions within the derived maps of longitudinal change by applying sparse canonical correlation analysis for neuroanatomy (SCCAN), as in [1]. SCCAN solves a constrained optimization problem that finds projection vectors, here represented as functions of $\mathrm{x}$ in the template image domain, that maximize the relationship between paired modalities/measurements as in figure2. In this study, we seek strictly positive projection vectors $\omega_{T}(\mathbf{x}) \geq 0, \omega_{J}(\mathbf{x}) \geq 0$ with $\left\|\omega_{T}\right\|_{1} \leq C_{T},\left\|\omega_{J}\right\|_{1} \leq C_{F}$ where $C$ is a constant related to the desired sparseness imposed by the $\mathrm{L} 1$ penalty, $\|\cdot\|_{1}$. The positivity constraint guarantees that both projection vectors, $\omega_{T, J}$, will have positive entries and may thus be viewed as a weighted average of the data. The L1 penalty guarantees that only a subset of voxels will enter the model. Under this formulation, SCCAN will maximize:

$$
\operatorname{argmax}\left(\omega_{T}, \omega_{J}\right): \operatorname{Corr}(\underbrace{\mathbf{T} \omega_{T}}_{\text {Thickness Projections }}, \underbrace{\mathbf{J} \omega_{J}}_{\text {Jacobian Projections }})-\lambda_{T}\left\|\omega_{T}\right\|_{1}-\lambda_{J}\left\|\omega_{J}\right\|_{1},
$$

where $\mathbf{T}$ is a matrix with columns containing voxels from the $\beta_{T}$ images and $n$ subjects number of rows, where $\mathbf{J}$ is a matrix with columns containing voxels from the $\beta_{J}$ images and $n$-subjects number of rows, Corr computes Pearson correlation and the $\lambda$ are inversely related to the sparseness costs, $C$. We apply SCCAN to determine regions that have significant covariation between the white matter Jacobian change and thickness change, with a sparseness value that selects a small, informative subset of both white matter and cortex (approximately 20\% of each). While both measures may be noisy, we expect the noisy components to be less well correlated than those that show robust, related change.

Note that steps $1-6$ do not use diagnosis in any way. The last variable selection step (6) only keeps those voxels that provide maximal information between the two 
measurements (here, thickness change and Jacobian change). Thus, if the covariation identified by SCCAN relates to unique disease patterns, detection power will be increased in the supervised, groupwise analysis, by removing uninformative background noise. The retained voxels in both modalities are then assessed by ANCOVA where age and sex effects are factored out to determine the significance of the disease alone.

\section{Results}

Evaluation 1: Sanity Check. We pass pseudo-longitudinal data to the SUAAV algorithm where three identical images are passed in and treated as if they were collected over three years. If there is any inconsistency in the implementation of the SUAAV pre-processing steps, illustrated in figure 1 SUAAV will yield a non-zero change in thickness and/or Jacobian. As expected, we gained zero differences in segmentation and thickness.

Evaluation 2: Test-Retest. We pass short interval longitudinal data to the SUAAV algorithm where four subjects underwent repeat imaging within one month of the first image. In this case, we use the cortical parcellations gained in our pipeline to provide 32 sample points for each image. We average the cortical thickness within each region over each time point. This approach mirrors that taken in [3] where Fischl and Dale computed a standard deviation of $0.25 \mathrm{~mm}$ in thickness measurements collected in short-interval repeat sessions. SUAAV produced a $0.1 \mathrm{~mm}$ standard deviation across these images, indicating a high level of repeatability, given that the image resolution is approximately $1 \mathrm{~mm}^{3}$. We also used Monte Carlo simulation to determine if our test-retest distribution of thickness change differs from a randomly sampled Gaussian distribution centered at zero with the same standard deviation $(0.104 \mathrm{~mm})$ and same number of samples $(4 * 32=128)$. We performed over 10,000 simulations where, for each simulation, we performed a t-test of the difference between our distribution and the random Gaussian sampling. 95.2\% of the results showed no difference, thus there is less than a five percent chance that the distribution is not Gaussian and centered at zero. In addition, we performed a Pearson chi-square normality test $(\mathrm{p}=0.2734)$ which also showed our test-retest distribution is close to normal.

Evaluation 3: Application to FTD-Elderly Longitudinal Change. Given the results of Evaluation 1 and 2, we test whether SUAAV is able to detect neurobiological effects of dementia expected to occur within the frontal and/or temporal lobes, as in FTD. Thus, the full SUAAV pipeline (with SCCAN) is used to process 26 FTD subjects and 7 elderly subjects. The SCCAN variable selection is used to restrict the ANCOVA test that uses a model of the form: Thickness Change $\approx x_{0}+$ age + gender + diagnosis. The same model is used for the Jacobian. The p-values associated with the diagnosis predictor are corrected by the false discovery rate and shown, rendered on the template cortex, in figure 3 . Middle frontal gyrus (MFG) and inferior frontal gyrus (IFG) on the left survives correction, along with anterior frontal lobe white matter. To eliminate the possibility that misregistration led to these results, we also interrogated the average thickness change across the MFG and IFG regions gained from the parcellation. These regional measures-which do not suffer from interpolation or registration error-also showed significant reduction over time in FTD relative to controls, thus affirming our voxel-wise findings. 


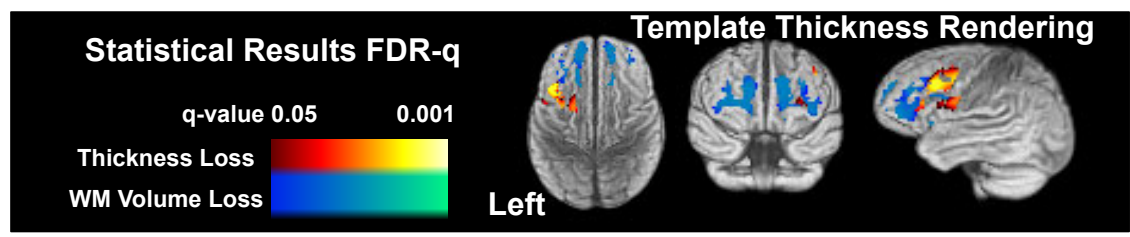

Fig. 3. The SUAAV method provides a restricted set of regions over which to perform statistical testing. Results are FDR corrected where we accept q-value (corrected p-value) $<0.05$ as significant with cluster size $>100 \mathrm{~mm}^{3}$. Left frontal cortex atrophies at a greater rate (approximately 6-10 \% per year) in FTD than controls. The template labeling identifies the cortical regions as middle frontal and inferior frontal gyrus. The white matter loss occurs bilaterally in the anterior frontal lobe. These findings are consistent with what is known about frontotemporal dementia.

\section{Discussion}

We presented an unbiased method for analyzing group differences in annualized cortical thickess and white matter volume change in dementia. Both unbiased image registration and segmentation algorithms were used. We showed that SUAAV produces zero change when we passed false serial data that contains identical images across time and produces zero-centered Gaussian distributed estimates of change across the brain when SUAAV is passed test-retest data from scanning sessions from the same subject imaged within a month. Finally, we showed that SUAAV produced significant, biologically plausible estimates of cortical and white matter atrophy within left, frontal cortex in FTD that differed from controls.

A critical choice when applying SUAAV is the sparseness penalty term. This term controls the number of voxels that enters into the downstream ANCOVA. The benefit is a vast reduction in the problem of multiple comparisons but this comes at the cost of a loss of detection power in regions that are rejected in this step. Specifically, in our study, regions of cortex that do not relate to white matter change (in the within-subject sense) will not enter into the testing procedure and vice versa. Additionally, the sparseness term should be selected based on a power analysis and a priori anatomical knowledge.

SUAAV provides a powerful new way to convert standard, serially collected MRI datasets into a pair of independent but covarying measurements that may be used to identify repeatable and reliable spatial changes in cortical thickness. We also sought to show that SUAAV provides a neuronatomically valid and meaningful way to increase power for longitudinal atrophy studies. While we have taken a high-level approach to evaluation of the full SUAAV pipeline, additional effort will be required to understand, in detail, the criticality of each step in SUAAV. Additionally, a deep understanding of parameter selection and testing on other datasets will be required to verify broad applicability of the algorithm.

\section{References}

1. Avants, B.B., Yushkevich, P., Pluta, J., Minkoff, D., Korczykowski, M., Detre, J., Gee, J.C.: The optimal template effect in hippocampus studies of diseased populations. Neuroimage (2010) (in press) 
2. Das, S.R., Avants, B.B., Grossman, M., Gee, J.C.: Registration based cortical thickness measurement. Neuroimage 45(3), 867-879 (April 2009), http://dx.doi.org/10.1016/j.neuroimage.2008.12.016

3. Fischl, B., Dale, A.M.: Measuring the thickness of the human cerebral cortex from magnetic resonance images. Proc. Natl. Acad. Sci. USA 97(20), 11050-11055 (2000), http://dx.doi.org/10.1073/pnas.200033797

4. Klein, A., Andersson, J., Ardekani, B.A., Ashburner, J., Avants, B., Chiang, M.C., Christensen, G.E., Collins, D.L., Gee, J., Hellier, P., Song, J.H., Jenkinson, M., Lepage, C., Rueckert, D., Thompson, P., Vercauteren, T., Woods, R.P., Mann, J.J., Parsey, R.V.: Evaluation of 14 nonlinear deformation algorithms applied to human brain MRI registration. Neuroimage 46(3), 786-802 (2009),

http://dx.doi.org/10.1016/j.neuroimage.2008.12.037

5. Klein, A., Ghosh, S.S., Avants, B., Yeo, B.T.T., Fischl, B., Ardekani, B., Gee, J.C., Mann, J.J., Parsey, R.V.: Evaluation of volume-based and surface-based brain image registration methods. Neuroimage (February 2010), http://dx.doi.org/10.1016/j.neuroimage.2010.01.091

6. Knight, W.D., Kim, L.G., Douiri, A., Frost, C., Rossor, M.N., Fox, N.C.: Acceleration of cortical thinning in familial alzheimer's disease. Neurobiol. Aging (December 2009), http://dx.doi.org/10.1016/j.neurobiolaging.2009.11.013

7. Kriegeskorte, N., Simmons, W.K., Bellgowan, P.S.F., Baker, C.I.: Circular analysis in systems neuroscience: the dangers of double dipping. Nat. Neurosci. 12(5), 535-540 (2009), http://dx.doi.org/10.1038/nn.2303

8. Krueger, C.E., Dean, D.L., Rosen, H.J., Halabi, C., Weiner, M., Miller, B.L., Kramer, J.H.: Longitudinal rates of lobar atrophy in frontotemporal dementia, semantic dementia, and Alzheimer's disease. Alzheimer Dis. Assoc. Disord. (June 2009), http://dx.doi.org/10.1097/WAD.0b013e3181a6f101

9. Scahill, R.I., Schott, J., Stevens, J.: Mapping the evolution of regional atrophy in Alzheimer's disease: unbiased analysis of fluid-registered serial MRI. Proc. Natl. Acad. Sci. USA 99, 4135-4137 (2002)

10. Shaw, P., Lalonde, F., Lepage, C., Rabin, C., Eckstrand, K., Sharp, W., Greenstein, D., Evans, A., Giedd, J.N., Rapoport, J.: Development of cortical asymmetry in typically developing children and its disruption in attention-deficit/hyperactivity disorder. Arch. Gen. Psychiatry 66(8), 888-896 (2009), http://dx.doi.org/10.1001/archgenpsychiatry.2009.103

11. Tustison, N.J., Gee, J.C.: N4ITK: Nick's N3 ITK implementation for MRI bias field correction. Insight Journal (2009)

12. Whitwell, J.L., Jack, C.R., Pankratz, V.S., Parisi, J.E., Knopman, D.S., Boeve, B.F., Petersen, R.C., Dickson, D.W., Josephs, K.A.: Rates of brain atrophy over time in autopsy-proven frontotemporal dementia and Alzheimer disease. Neuroimage 39(3), 1034-1040 (2008), http://dx.doi.org/10.1016/j.neuroimage.2007.10.001

13. Whitwell, J.L., Jack, C.R., Parisi, J.E., Knopman, D.S., Boeve, B.F., Petersen, R.C., Ferman, T.J., Dickson, D.W., Josephs, K.A.: Rates of cerebral atrophy differ in different degenerative pathologies. Brain 130(Pt. 4), 1148-1158 (2007),

http://dx.doi.org/10.1093/brain/awm021

14. Yushkevich, P.A., Avants, B.B., Das, S.R., Pluta, J., Altinay, M., Craige, C., Initiative, A.D.N.: Bias in estimation of hippocampal atrophy using deformation-based morphometry arises from asymmetric global normalization: an illustration in adni $3 \mathrm{t}$ mri data. Neuroimage 50(2), 434-445 (2010) 